\title{
Mass in Lovelock unique vacuum gravity theories
}

\author{
Gabriel Arenas-Henriquez $\odot,{ }^{1, *}$ Robert B. Mann, ${ }^{2,3, \uparrow}$ Olivera Miskovic $\odot{ }^{4, \$}$ and Rodrigo Olea ${ }^{1, \S}$ \\ ${ }^{1}$ Departamento de Ciencias Físicas, Universidad Andres Bello, Sazié 2212, Piso 7, Santiago, Chile \\ ${ }^{2}$ Department of Physics and Astronomy, University of Waterloo, Waterloo, Ontario N2L 3G1, Canada \\ ${ }^{3}$ Perimeter Institute, 31 Caroline Street North, Waterloo, Ontario N2L 2Y5, Canada \\ ${ }^{4}$ Instituto de Física, Pontificia Universidad Católica de Valparaíso, Casilla 4059, Valparaíso, Chile
}

(Received 1 July 2019; published 23 September 2019)

\begin{abstract}
We derive an expression for conserved charges in Lovelock anti-de Sitter gravity for solutions having $k$-fold degenerate vacua, making manifest a link between the degeneracy of a given vacuum and the nonlinearity of the energy formula. We show for a black hole solution to the field equations on a branch of multiplicity $k$ that its mass comes from an expression that contains the product of $k$ Weyl tensors. We prove that all divergent contributions of the type (Weyl) $)^{q}$, with $1 \leq q<k$, are suppressed. Our conserved charge definition is a natural generalization of the conformal mass by Ashtekar, Magnon and Das to the cases when $k>1$. Our results provide insight on the holographic properties of degenerate Lovelock theories.
\end{abstract}

DOI: $10.1103 /$ PhysRevD.100.064038

\section{INTRODUCTION}

In the ongoing effort to construct an ultraviolet complete theory of quantum gravity, higher curvature theories of gravity emerge as corrections to the usual Einstein-Hilbert term. Such theories have now come to play an important role in cosmology, black hole physics, supergravity, string theory, and holography. They provide a framework for understanding which features of gravitational theory are generic and which are special. Amongst the most important of these theories is Lovelock gravity [1], which is the natural generalization of general relativity to $D$ dimensions in two important respects: its field equations are of secondorder and it is free of ghosts when expanded on a background of constant curvature [2].

One of the most important questions to address in any gravitational theory is the definition of conserved charges. This is a subtle issue in gravitational physics because of the equivalence principle, which makes localization of gravitational energy (and momentum and angular momentum) fraught with ambiguities [3]. Approaches for addressing this problem date back six decades [4,5] and more, and include a mixture of global [6-10] and quasilocal [11-15]

\footnotetext{
*gabriel.arenas.henriquez@gmail.com

rbmann@uwaterloo.ca

*olivera.miskovic@pucv.cl

rodrigo.olea@unab.cl
}

Published by the American Physical Society under the terms of the Creative Commons Attribution 4.0 International license. Further distribution of this work must maintain attribution to the author(s) and the published article's title, journal citation, and DOI. Funded by SCOAP. methods. Generalizations to Lovelock gravity have been carried out [16], and a universal form of the boundary term yielding a background-independent definition of conserved quantities for any Lovelock gravity theory with anti-de Sitter (AdS) asymptotics has been constructed [17].

The purpose of this paper is to extend our understanding of conserved charges in Lovelock gravity to degenerate solutions of the field equations. The simplest form of such solutions is that of a $k$-fold degenerate AdS vacuum. In general the field equations of $N$ th order Lovelock gravity admit as many as $N$ distinct vacua of constant curvature, each with their own effective cosmological constant. However these vacua need not be distinct-depending on the values of the Lovelock coupling constants, as many as $k$ cosmological constants can be equal to each other, where $k \leq N$; when this takes place we say that the vacuum is $k$-fold degenerate. This concept extends to solutions, such as black holes, that do not have constant curvature.

Understanding what the conserved charges are for such solutions is rather subtle. The asymptotic falloff conditions differ from those cases in which the solution has no degeneracy, and standard approaches [7-9] do not work.

In this paper we address this problem. We obtain an expression for conserved charges in Lovelock AdS gravity for $k$-fold degenerate solutions. In particular we demonstrate that the mass of a black hole solution to the field equations on a branch of multiplicity $k$ comes from an expression containing the product of $k$ Weyl tensors. There is thus a link between the degeneracy of a given vacuum and the nonlinearity of the energy formula. We find that potentially divergent contributions of the type (Weyl) ${ }^{q}$, with $1 \leq q<k$, are in fact suppressed. Noting the obstruction to linearization of such solutions about a constant 
curvature background [18,19], our results can provide useful insight on the holographic properties of degenerate Lovelock gravity. This may be regarded as a natural generalization of conformal mass to these theories [8,9].

The outline of our paper is as follows. We begin in Sec. II with a review of Lovelock gravity, highlighting the notion of degenerate solutions. In Sec. III we review the construction of conserved charges in the Kounterterm formalism [16], and in Sec. IV we derive a general formula for conserved charges for degenerate solutions (technical details are shown in Appendix A). Although even and odd dimensions need to be treated distinctly, we find that a single expression is valid in any dimension, and discuss its applications to obtaining the mass of a black hole solution. We summarize our work in Sec. V.

\section{LOVELOCK ADS GRAVITY}

Lovelock theory of gravity is the most general theory whose dynamics is described by a second-order equation of motion. The action on a $D$-dimensional manifold $\mathcal{M}$ endowed by the metric $g_{\mu \nu}$ is a polynomial in the Riemann curvature,

$$
I=\frac{1}{16 \pi G} \int_{\mathcal{M}} d^{D} x \sqrt{-g} \sum_{p=0}^{N} \alpha_{p} \mathcal{L}_{p}(R),
$$

where $\alpha_{p}$ are the coupling constants, $N=[(D-1) / 2]$ and $\mathcal{L}_{p}$ is the dimensional continuation of the $p$ th Euler density,

$$
\mathcal{L}_{p}=\frac{1}{2^{p}} \delta_{\nu_{1} \ldots \nu_{2 p}}^{\mu_{1} \ldots \mu_{2 p}} R_{\mu_{1} \mu_{2}}^{\nu_{1} \nu_{2}} \ldots R_{\mu_{2 p-1} \mu_{2 p}}^{\nu_{2 p-1} \nu_{2 p}}
$$

Here, $\delta_{\nu_{1} \ldots \nu_{m}}^{\mu_{1} \ldots \mu_{m}}=\operatorname{det}\left[\delta_{\nu_{1}}^{\mu_{1}} \ldots \delta_{\nu_{m}}^{\mu_{m}}\right]$ denotes a completely antisymmetric Kronecker delta of rank $m$. We can think of this series as a modification to the Einstein-Hilbert action with negative cosmological constant $\Lambda=-(D-1)(D-2) / 2 \ell^{2}$ with $\ell$ as the bare AdS radius. The Einstein-Hilbert term corresponds to the first two terms in the polynomial (2.1), with $\alpha_{0}=-2 \Lambda$ and $\alpha_{1}=1$. Then the variation of the action leads to the following equations of motion,

$$
\mathcal{E}_{\mu}^{\nu}=R_{\mu}^{\nu}-\frac{1}{2} R \delta_{\mu}^{\nu}+\Lambda \delta_{\mu}^{\nu}-H_{\mu}^{\nu}=0
$$

where $H_{\mu}^{\nu}$ is the generalized Lanczos tensor that contains all the higher-curvature contributions to the Einstein tensor in $D>4$,

$$
H_{\mu}^{\nu}=\sum_{p=2}^{N} \frac{\alpha_{p}}{2^{p+1}} \delta_{\mu \mu_{1} \ldots \mu_{2 p}}^{\nu \nu_{1} \ldots \nu_{2 p}} R_{\nu_{1} \nu_{2}}^{\mu_{1} \mu_{2}} \ldots R_{\nu_{2 p-1} \nu_{2 p}}^{\mu_{2 n-1} \mu_{2 p}}
$$

\section{A. Vacua of the theory}

An alternative way to write the field equations is

$$
\begin{aligned}
\mathcal{E}_{\mu}^{\nu}= & \sum_{p=0}^{N} \frac{\alpha_{p}}{2^{p+1}} \delta_{\mu \mu_{1} \ldots \mu_{2 p}}^{\nu \nu_{1} \ldots \nu_{2 p}}\left(R_{\nu_{1} \nu_{2}}^{\mu_{1} \mu_{2}}+\frac{1}{\ell_{\mathrm{eff}}^{(1) 2}} \delta_{\nu_{1} \nu_{2}}^{\mu_{1} \mu_{2}}\right) \\
& \ldots\left(R_{\nu_{2 p-1} \nu_{2 p}}^{\mu_{2 n-1} \mu_{2 p}}+\frac{1}{\ell_{\mathrm{eff}}^{(N) 2}} \delta_{\nu_{2 p-1} \nu_{2 p}}^{\mu_{2 n-1} \mu_{2 p}}\right) \\
= & 0,
\end{aligned}
$$

where $\ell_{\mathrm{eff}}^{(i)}$, with $i=1, \ldots, N$, are effective AdS radii. They differ from the bare AdS radius $\ell$ due to the contribution of higher-order terms through the couplings $\left\{\alpha_{2}, \ldots, \alpha_{N}\right\}$. Equation (2.5) explicitly shows that maximally-symmetric spacetimes are particular vacuum solutions of the theory, whose Riemann curvature is

$$
R_{\alpha \beta}^{\mu \nu}=-\frac{1}{\ell_{\mathrm{eff}}^{2}} \delta_{\alpha \beta}^{\mu \nu},
$$

which we can also write as $R+\frac{1}{\ell_{\text {eff }}^{2}} \delta^{[2]}=0$, suppressing indices using the square-bracket notation. We can find the form of $\ell_{\text {eff }}$ by inserting the above expression into $\mathcal{E}_{\mu}^{\nu}=0$ and obtain the characteristic polynomial of order $N$ in the variable $\ell_{\mathrm{eff}}^{-2}$,

$$
\Delta\left(\ell_{\mathrm{eff}}^{-2}\right) \equiv \sum_{p=0}^{N} \frac{(D-3) !(-1)^{p-1} \alpha_{p}}{(D-2 p-1) !}\left(\frac{1}{\ell_{\mathrm{eff}}^{2}}\right)^{p}=0 .
$$

The roots of $\Delta$ depend on the parameter set $\left\{\alpha_{p}\right\}$; the simplest case is $N=1$ with $\Lambda=-(D-1)(D-2) / 2 / \ell_{\mathrm{eff}}^{2}$, for which $\ell_{\text {eff }}=\ell$. Writing $\lambda=\ell_{\text {eff }}^{-2}$, if there are $N$ real roots $\lambda_{i}(i=1 \ldots N)$ then the theory has $N$ maximally symmetric vacua, which can be $\operatorname{AdS}\left(\lambda_{i}>0\right), \mathrm{dS}\left(\lambda_{i}<0\right)$ or flat $\left(\lambda_{i}=0\right)$. If a root $\lambda_{i}$ is complex then a maximally symmetric space for this value of $\ell_{\text {eff }}$ is not a particular solution of the theory.

We are interested in a spacetime that is asymptotically AdS, defined as a branch of the theory by the corresponding AdS radius $\ell_{\text {eff }}$. The root itself can be simple, or $k$-fold degenerate, describing a vacuum of multiplicity $k$.

The existence of a vacuum with multiplicity $k>1$ is a defining feature of degenerate theories. A particular root is simple if its characteristic polynomial satisfies

$$
\begin{aligned}
\Delta^{(1)}\left(\ell_{\mathrm{eff}}^{-2}\right) & =\frac{d \Delta}{d\left(\ell_{\mathrm{eff}}^{-2}\right)} \\
& =\sum_{p=1}^{N} \frac{(D-3) !(-1)^{p-1} p \alpha_{p}}{(D-2 p-1) !}\left(\frac{1}{\ell_{\mathrm{eff}}^{2}}\right)^{p-1} \neq 0 .
\end{aligned}
$$

The relation $\Delta^{(1)}=0$ defines a critical point in the parameter space, which acts as an obstruction to the linearization of the theory (see, e.g., Ref. [18] in the context of 
Einstein-Gauss-Bonnet gravity and Ref. [19] in Lovelock gravity). This fact prevents the obtention of an energy formula as a linearized charge $[20,21]$. The nature of this criticality will depend on how degenerate the particular branch is.

We will focus on a theory with a vacuum that is $k$-fold degenerate. It is defined by the fact that all derivatives of $\Delta$ vanish up to order $k$,

$$
\begin{aligned}
& \Delta^{(q)}\left(\ell_{\mathrm{eff}}^{-2}\right)=\frac{1}{q !} \frac{d^{q} \Delta}{d\left(\ell_{\mathrm{eff}}^{-2}\right)^{q}}=0, \quad \text { when } 1 \leq q \leq k-1, \\
& \Delta^{(k)}\left(\ell_{\mathrm{eff}}^{-2}\right)=\sum_{p=k}^{N}\left(\begin{array}{l}
p \\
k
\end{array}\right) \frac{(D-3) !(-1)^{p-1} \alpha_{p}\left(\ell_{\mathrm{eff}}^{-2}\right)^{p-k}}{(D-2 p-1) !} \neq 0 .
\end{aligned}
$$

The above condition means that the equations of motion (2.5) are factorizable by $\left(R+\frac{1}{\ell_{\mathrm{eff}}^{2}} \delta^{[2]}\right)^{k}$.

Examples of degenerate theories with maximal multiplicity are, in even dimensions $D=2 n$, Born-Infeld AdS gravity,

$\alpha_{p}^{\mathrm{BI}}=\left(\begin{array}{c}n-1 \\ p\end{array}\right) \frac{2^{n-2}(2 n-2 p-1) !}{\ell^{2(n-p-1)}}, \quad 0 \leq p \leq n-1$,

and, in odd dimensions $D=2 n+1$, Chern-Simons AdS gravity,

$$
\alpha_{p}^{\mathrm{CS}}=\left(\begin{array}{c}
n \\
p
\end{array}\right) \frac{2^{n-1}(2 n-2 p) !}{\ell^{2(n-p)}}, \quad 0 \leq p \leq n .
$$

A special feature of Chern-Simons AdS gravity is that it has enhanced local symmetry from Lorentz to AdS, so its dynamics and a number of degrees of freedom are different compared to any other Lovelock gravity. In that sense, Chern-Simons AdS gravity is a truly gauge theory. Similar features hold for Chern-Simons de Sitter and Poincaré gravities.

A generalization of the above theories, which considers a unique solution for $\ell_{\text {eff }}$ with an intermediate degeneracy $k$, is the so-called Lovelock Unique Vacuum (LUV) AdS theory in $D$ dimensions. Here, $\Delta^{(k)}$ is the only nonvanishing coefficient meaning that, apart from Eq. (2.9), there is also the additional requirement $\Delta^{(q)}\left(\ell_{\text {eff }}^{-2}\right)=0$ when $k<q \leq N$. In this case, the coupling constants have the form

$$
\begin{aligned}
& \alpha_{p}^{\mathrm{LUV}}=\left(\begin{array}{l}
k \\
p
\end{array}\right) \frac{2^{k-1}(D-2 p-1) !}{\ell^{2(k-p)}} 0 \leq p \leq k, \\
& \alpha_{p>k}^{\mathrm{LUV}}=0 .
\end{aligned}
$$

Einstein-Hilbert, Born-Infeld and Chern-Simons AdS gravities are particular cases of LUV AdS theories.

Our present goal is to find a mass formula for all those gravity theories where the degeneracy is higher than one in odd and even spacetime dimensions. To this end, we have to analyze the asymptotic behavior of the corresponding solutions. In the next section, we obtain the falloff of the relevant quantities for static geometries with $1 \leq k \leq N$.

\section{B. Asymptotic behavior of Lovelock AdS solutions}

A topological, static black hole solution in the local coordinates $x^{\mu}=\left(t, r, y^{m}\right)(m=2, \ldots, D-1)$ is described by the line element

$$
d s^{2}=-f(r) d t^{2}+\frac{1}{f(r)} d r^{2}+r^{2} \gamma_{m n}(y) d y^{m} d y^{n},
$$

where $\gamma_{n m}$ is the metric of the transverse section of constant curvature $\kappa=+1,0,-1$. The metric function $f(r)$ is given by the algebraic master equation obtained as the first integral of the equations of motion [22-24],

$$
\sum_{p=0}^{N} \frac{\alpha_{p}(D-3) !}{(D-2 p-1) !}\left(\frac{\kappa-f(r)}{r^{2}}\right)^{p}=\frac{\mu}{r^{D-1}},
$$

where $\mu$ is an integration constant related to the mass of the solution, as we shall discuss below. In the case of asymptotically AdS spaces, $f(r)$ behaves as

$$
f(r)=\kappa+\frac{r^{2}}{\ell_{\mathrm{eff}}^{2}}+\epsilon(r),
$$

where $f_{\mathrm{AdS}}=\kappa+\frac{r^{2}}{\ell_{\mathrm{eff}}^{2}}$ is the global AdS space and $\epsilon(r)$ is a function that, in most cases, decays sufficiently fast. Plugging this into the master equation and expanding in the asymptotic region $(1 / r \rightarrow 0)$, we find

$$
\begin{aligned}
\frac{\mu}{r^{D-1}} & =\sum_{p=0}^{N} \frac{(-1)^{p}(D-3) ! \alpha_{p}}{(D-2 p-1) !}\left(\frac{1}{\ell_{\mathrm{eff}}^{2}}+\frac{\epsilon}{r^{2}}\right)^{p} \\
& =\sum_{q=0}^{N} \sum_{p=q}^{N} \frac{(-1)^{p}(D-3) ! \alpha_{p}}{(D-2 p-1) !}\left(\begin{array}{c}
p \\
q
\end{array}\right)\left(\frac{1}{\ell_{\mathrm{eff}}^{2}}\right)^{p-q}\left(\frac{\epsilon}{r^{2}}\right)^{q},
\end{aligned}
$$

or equivalently

$$
\frac{\mu}{r^{D-1}}=\sum_{q=0}^{N} \Delta^{(q)}\left(\frac{\epsilon}{r^{2}}\right)^{q},
$$

where $\Delta^{(q)}$ is given by Eq. (2.9). We can expand the above series knowing that, for a $k$-fold degenerate vacuum, the first nonvanishing term is $\Delta^{(k)}$ yielding 


$$
\frac{\mu}{r^{D-1}}=\Delta^{(k)}\left(\frac{\epsilon}{r^{2}}\right)^{k}+\Delta^{(k+1)}\left(\frac{\epsilon}{r^{2}}\right)^{k+1}+\cdots
$$

Furthermore, if $\Delta^{(k+1)}=0$, it can be shown that $\Delta^{(q)}=0$ for all $q>k$. Then $\epsilon(r)$ can be solved exactly as $\epsilon=\left(\frac{\mu}{\Delta^{(k)} r^{D-2 k-1}}\right)^{1 / k}$.

For an arbitrary value of $\Delta^{(k+1)}$, we can assume an asymptotic behavior for $\epsilon(r)$ of the form

$\epsilon(r)=\frac{A}{r^{x}}+\frac{B}{r^{y}}+\cdots, \quad y>x \geq 0, \quad A \neq 0$,

where $A, B, x$, and $y$ are coefficients to be determined. Inserting Eq. (2.19) into (2.18), we obtain

$$
\begin{aligned}
\frac{\mu}{r^{D-1}}= & \Delta^{(k)}\left(\frac{A^{k}}{r^{(x+2) k}}+\frac{k A^{k-1} B}{r^{(x+2)(k-1)+y+2}}+\cdots\right) \\
& +\Delta^{(k+1)}\left(\frac{A^{k+1}}{r^{(x+2)(k+1)}}+\cdots\right) .
\end{aligned}
$$

At leading order, we find

$$
\frac{\mu}{r^{D-1}}=\Delta^{(k)} \frac{A^{k}}{r^{(x+2) k}}
$$

and therefore

$$
x=\frac{D-2 k-1}{k}, \quad A=\left(\frac{\mu}{\Delta^{(k)}}\right)^{\frac{1}{k}} .
$$

Notice that the mass parameter may be negative and that $A$ can have any sign for even $k$. The subleading contributions become

$$
\begin{aligned}
0= & \Delta^{(k)}\left(\frac{k A^{k-1} B}{r^{(x+2)(k-1)+y+2}}+\cdots\right) \\
& +\Delta^{(k+1)}\left(\frac{A^{k+1}}{r^{(x+2)(k+1)}}+\cdots\right) .
\end{aligned}
$$

We can distinguish three cases for the coefficients $A$ and $B$ :

(a) When $A=B=0$, the solution becomes the vacuum state of the theory, $\mu=0$, i.e., global AdS.

(b) When $A \neq 0$ and $B=0$, the solution exists only if $\Delta^{(k+1)}=0$. As discussed after Eq. (2.18), $\Delta^{(k)}$ is the only nonvanishing coefficient and $\epsilon$ can be solved exactly. This case is that of LUV theories.

(c) When $A, B \neq 0$, the terms along $\Delta^{(k)}$ and $\Delta^{(k+1)}$ are of the same order. That implies

$$
y=\frac{2(D-1)-2 k}{k}
$$

and, in turn, the coefficient $B$ reads

$$
B=-\frac{\Delta^{(k+1)} A^{2}}{k \Delta^{(k)}}=-\frac{\Delta^{(k+1)}}{k \Delta^{(k)}}\left(\frac{\mu}{\Delta^{(k)}}\right)^{\frac{2}{k}} .
$$

The cases (a)-(c) are covered by the formulas (2.2), (2.24) and (2.25). In this way, for a degenerate vacuum with multiplicity $k$, we find a general falloff of the metric function as

$$
\begin{aligned}
f(r)= & \kappa+\frac{r^{2}}{\ell_{\mathrm{eff}}^{2}}+\left(\frac{\mu}{\Delta^{(k)} r^{D-2 k-1}}\right)^{\frac{1}{k}} \\
& -\frac{\Delta^{(k+1)}}{k \Delta^{(k) 2}}\left(\frac{\mu^{2}}{\Delta^{(k)} r^{2(D-1)-2 k}}\right)^{\frac{1}{k}}+\cdots
\end{aligned}
$$

We illustrate the above relation with the exact solutions in LUV gravity for $k<N$ [25]

$$
f_{\mathrm{LUV}}(r)=\kappa+\frac{r^{2}}{\ell_{\mathrm{eff}}^{2}}-\left(\frac{2 G M}{r^{D-2 k-1}}\right)^{\frac{1}{k}}
$$

where $G$ is the gravitational constant. In maximally degenerate cases, these are Chern-Simons and BornInfeld AdS black holes

$$
\begin{aligned}
& f_{\mathrm{CS}}(r)=\kappa+\frac{r^{2}}{\ell_{\mathrm{eff}}^{2}}-(2 G M+1)^{\frac{1}{n}} \\
& f_{\mathrm{BI}}(r)=\kappa+\frac{r^{2}}{\ell_{\mathrm{eff}}^{2}}-\left(\frac{2 G M}{r}\right)^{\frac{1}{n-1}}
\end{aligned}
$$

The mass parameter in the Chern-Simons case has been redefined so that the horizon shrinks to a point when $M \rightarrow 0$. This produces a mass gap, $M=-1 / 2 G$, between the Chern-Simons black hole, $M \geq 0$, and global AdS space with $M=-1 / 2 G$.

It is worthwhile noting that the identification of the mass parameter $M$ [related to the integration constant $\mu$ in the general formula (2.26)] as the total mass of the black hole was made in Ref. [25] and, for $k=2$, in Ref. [18], based on thermodynamic calculations. In Ref. [25], it has been also obtained as a Hamiltonian mass in the minisuperspace approach. In our method, however, we calculate the mass as the one that comes from Noether theorem, once the action has been supplemented by adequate boundary terms. This notion agrees with the thermodynamic and Hamiltonian mass.

In what follows, we make extensive use of the falloff of the metric (2.26) to find both the AdS curvature and the corresponding Weyl tensor.

\section{AdS curvature and Weyl tensor}

The only nonvanishing components of the Riemann tensor for the static solution (2.13) are 


$$
\begin{aligned}
R_{t r}^{t r} & =-\frac{1}{2} f^{\prime \prime}, \quad R_{r m}^{r n}=R_{t m}^{t n}=-\frac{f^{\prime}}{2 r} \delta_{m}^{n}, \\
R_{m_{1} m_{2}}^{n_{1} n_{2}} & =\frac{\kappa-f}{r^{2}} \delta_{m_{1} m_{2}}^{n_{1} n_{2}} .
\end{aligned}
$$

Using the falloff (2.26), we get

$$
\begin{aligned}
R_{t r}^{t r}= & -\frac{1}{\ell_{\mathrm{eff}}^{2}}-\frac{(D-2 k-1)(D-k-1)}{2 k^{2}}\left(\frac{\mu}{\Delta^{(k)} r^{D-1}}\right)^{\frac{1}{k}} \\
& +\mathcal{O}\left(r^{-\frac{2(D-1)}{k}}\right), \\
R_{r m}^{r n}= & R_{t m}^{t n}=\left[-\frac{1}{\ell_{\mathrm{eff}}^{2}}+\frac{D-2 k-1}{2 k}\left(\frac{\mu}{\Delta^{(k)} r^{D-1}}\right)^{\frac{1}{k}}\right] \delta_{m}^{n} \\
& +\mathcal{O}\left(r^{-\frac{2(D-1)}{k}}\right), \\
R_{m_{1} m_{2}}^{n_{1} n_{2}}= & {\left[-\frac{1}{\ell_{\mathrm{eff}}^{2}}+\left(\frac{\mu}{\Delta^{(k)} r^{D-1}}\right)^{\frac{1}{k}}\right] \delta_{m_{1} m_{2}}^{n_{1} n_{2}}+\mathcal{O}\left(r^{-\frac{2(D-1)}{k}}\right) . }
\end{aligned}
$$

Furthermore, the AdS curvature

$$
F_{\alpha \beta}^{\mu \nu}=R_{\alpha \beta}^{\mu \nu}+\frac{1}{\ell_{\mathrm{eff}}^{2}} \delta_{\alpha \beta}^{\mu \nu},
$$

is the only part of the field strength of the local $S O(D-1,2)$ group that differs from zero in a Riemannian geometry. Using Eqs. (2.30), it is straightforward to evaluate it as

$$
\begin{aligned}
F_{t r}^{t r}= & -\frac{(D-2 k-1)(D-k-1)}{2 k^{2}}\left(\frac{\mu}{\Delta^{(k)} r^{D-1}}\right)^{\frac{1}{k}} \\
& +\mathcal{O}\left(r^{-\frac{2(D-1)}{k}}\right), \\
F_{r m}^{r n}= & F_{t m}^{t n}=\frac{D-2 k-1}{2 k}\left(\frac{\mu}{\Delta^{(k)} r^{D-1}}\right)^{\frac{1}{k}} \delta_{m}^{n}+\mathcal{O}\left(r^{-\frac{2(D-1)}{k}}\right), \\
F_{m_{1} m_{2}}^{n_{1} n_{2}}= & \left(\frac{\mu}{\Delta^{(k)} r^{D-1}}\right)^{\frac{1}{k}} \delta_{m_{1} m_{2}}^{n_{1} n_{2}}+\mathcal{O}\left(r^{-\frac{2(D-1)}{k}}\right) .
\end{aligned}
$$

We can express the above in a useful way in terms of the Weyl tensor, which is defined in terms of the Riemann tensor and its contractions as

$W_{\alpha \beta}^{\mu \nu}=R_{\alpha \beta}^{\mu \nu}-\frac{1}{D-2} \delta_{[\alpha}^{[\mu} R_{\beta]}^{\nu]}+\frac{R}{(D-1)(D-2)} \delta_{\alpha \beta}^{\mu \nu}$,

where the second term is the skew-symmetric product between the Kronecker delta and the Ricci tensor, $\delta_{[\alpha}^{[\mu} R_{\beta]}^{\nu]}=$ $\delta_{\alpha}^{\mu} R_{\beta}^{\nu}-\delta_{\alpha}^{\nu} R_{\beta}^{\mu}-\delta_{\beta}^{\mu} R_{\alpha}^{\nu}+\delta_{\beta}^{\nu} R_{\alpha}^{\mu}$. In Einstein AdS gravity, the on-shell Weyl tensor coincides with the AdS curvature. However in Lovelock AdS gravity the higher-order contributions modify the above relation such that the on-shell Weyl tensor can be written as

$$
W_{\alpha \beta}^{\mu \nu}=F_{\alpha \beta}^{\mu \nu}+X_{\alpha \beta}^{\mu \nu},
$$

where the tensor $X_{\alpha \beta}^{\mu \nu}$ is constructed from the generalized Lanczos tensor (2.4) and its trace $H=H_{\mu}^{\mu}$,

$$
X_{\alpha \beta}^{\mu \nu}=\left(\frac{1}{\ell^{2}}-\frac{1}{\ell_{\mathrm{eff}}^{2}}+\frac{2 H}{(D-1)(D-2)}\right) \delta_{\alpha \beta}^{\mu \nu}-\frac{1}{D-2} \delta_{[\alpha}^{[\mu} H_{\beta]}^{\nu]} .
$$

A computation based on the falloff of the Riemann tensor (2.30) computed above shows that the components of $X$ are always subleading in $r$ with respect to the AdS curvature. More precisely, $X_{\alpha \beta}^{\mu \nu}=\mathcal{O}\left(\mu^{2 / k} / r^{2(D-1) / k}\right)$ asymptotically. Therefore, the leading order of the AdS curvature and the Weyl tensor is

$$
W_{\alpha \beta}^{\mu \nu}=F_{\alpha \beta}^{\mu \nu}=\mathcal{O}\left(\mu^{1 / k} / r^{(D-1) / k}\right) .
$$

In the next section we show that, for a given theory with degeneracy $k$, the conserved charge formula is proportional to the $k$ th power of the Weyl tensor. This is obtained as a consistent truncation of the charges found in Refs. [17,26] in order to produce a finite energy flux at the asymptotic region.

\section{KOUNTERTERMS AND CONSERVED CHARGES}

The Lovelock AdS action is infrared (IR) divergent and has to be renormalized by adding boundary counterterms. Instead of obtaining the local counterterm series perturbatively, as in standard holographic renormalization [7,27-29], the idea behind the Kounterterm method $[16,30]$ is that the bulk action is supplemented with an appropriate boundary term that is linked either to topological invariants or ChernSimons forms. In $D=d+1$ dimensions, the renormalized action defined on the manifold $\mathcal{M}$ reads

$$
I_{\text {ren }}=I_{\text {bulk }}+c_{d} \int_{\partial \mathcal{M}} d^{d} x B_{d}(h, K, \mathcal{R}),
$$

where $B_{d}(h, K, \mathcal{R})$ is a scalar density on the boundary $\partial \mathcal{M}$ that depends on the boundary metric, the extrinsic curvature, and the boundary curvature. The overall factor $c_{d}$ is a given coupling. It has been shown that the method appropriately leads to finite conserved charges in highercurvature gravity theories [26,31] and it is also useful for computing holographic quantities such as entanglement entropy [32].

As usual, the charges are expressed as an integral over a co-dimension 2 surface at fixed time and radial infinity. We can proceed taking a radial foliation of the spacetime $\mathcal{M}$ in Gauss-normal coordinates,

$$
d s^{2}=N^{2}(r) d r^{2}+h_{i j}(r, x) d x^{i} d x^{j},
$$


where $N(r)$ is the lapse function and $h_{i j}$ is the induced metric at a fixed $r$. In turn, the boundary metric admits a time-like ADM foliation as

$$
h_{i j} d x^{i} d x^{j}=-\tilde{N}^{2}(t) d t^{2}+\sigma_{m n}\left(d y^{m}+\tilde{N}^{m} d t\right)\left(d y^{n}+\tilde{N}^{n} d t\right),
$$

where now $\sqrt{-h}=\tilde{N} \sqrt{\sigma}$, with $\sigma_{m n}$ the co-dimension two metric of the asymptotic boundary $\Sigma_{\infty}$. The unit normal to the hypersurface is given by $u_{j}=\left(u_{t}, u_{m}\right)=(-\tilde{N}, 0)$ and therefore, the conserved charges are given by the surface integral

$$
Q[\xi]=\int_{\Sigma_{\infty}} d^{d-1} y \sqrt{\sigma} u_{j} \xi^{i}\left(\tau_{i}^{j}+\tau_{(0) i}^{j}\right),
$$

where $\xi^{i}$ is an asymptotic Killing vector. The charge density tensor is naturally split in two contributions: $\tau_{i}^{j}$ that, when integrated, can be identified with the mass and angular momentum of the black hole, and $\tau_{(0) i}^{j}$ is associated with the vacuum/Casimir energy in the context of AdS/CFT correspondence [7].

In even dimensions $D=2 n, \tau_{i}^{j}$ has the form [26]

$$
\begin{aligned}
\tau_{i}^{j}= & \frac{1}{2^{n-2}} \delta_{i_{1} i_{2} \ldots i_{2 n-1}}^{j j_{2} \ldots j_{2 n-1}} K_{i}^{i_{1}}\left[\frac{1}{16 \pi G} \sum_{p=1}^{n-1} \frac{p \alpha_{p}}{(2 n-2 p) !} R_{j_{2} j_{3}}^{i_{2} i_{3}} \ldots R_{j_{2 p-2} j_{2 p-1}}^{i_{2 p} i_{2 p-1}}\right. \\
& \left.\times \delta_{j_{2 p} j_{2 p+1}}^{i_{2} i_{2} i_{2 n+1}} \ldots \delta_{j_{2 n-2} j_{2 n-1}}^{i_{2 n-2} i_{2 n-1}}+n c_{2 n-1} R_{j_{2} j_{3}}^{i_{2} i_{3}} \ldots R_{j_{2 n-2} j_{2 n-1}}^{i_{2 n-2} i_{2 n-1}}\right],
\end{aligned}
$$

and $\tau_{(0) i}^{j}=0$. The coupling in (3.1) is fixed from the action principle,

$$
c_{2 n-1}=-\frac{1}{16 \pi n G} \sum_{p=1}^{n-1} \frac{p \alpha_{p}}{(D-2 p) !}\left(-\ell_{\mathrm{eff}}^{2}\right)^{n-p} .
$$

In odd dimensions $D=2 n+1$, the charge density tensor reads

$$
\begin{aligned}
\tau_{i}^{j}= & \frac{1}{2^{n-2}} \delta_{i_{1} i_{2} \ldots i_{2 n}}^{j j_{2} \ldots j_{2 n}} K_{i}^{i_{1}} \delta_{j_{2}}^{i_{2}}\left[\frac{1}{16 \pi G} \sum_{p=1}^{n} \frac{p \alpha_{p}}{(2 n-2 p+1) !} R_{j_{3} j_{4}}^{i_{3} i_{4}}\right. \\
& \ldots R_{j_{2 p-1} j_{2 p}}^{i_{2 p-1} i_{2 p}} \delta_{j_{2 p+1} j_{2 p+2}}^{i_{2 p+1} i_{2 p+2}} \\
& \cdots \delta_{j_{2 n-1} j_{2 n}}^{i_{2 n-1} i_{2 n}}+n c_{2 n} \int_{0}^{1} d u\left(R_{j_{3} j_{4}}^{i_{3} i_{4}}+\frac{u^{2}}{\ell_{\mathrm{eff}}^{2}} \delta_{j_{3} j_{4}}^{i_{3} i_{4}}\right) \\
& \left.\ldots\left(R_{j_{2 n-1} j_{2 n}}^{i_{2 n-1} i_{2 n}}+\frac{u^{2}}{\ell_{\mathrm{eff}}^{2}} \delta_{j_{2 n-1} j_{2 n}}^{i_{2 n-1} i_{2 n}}\right)\right]
\end{aligned}
$$

and

$$
\begin{aligned}
\tau_{(0) i}^{j}= & -\frac{n c_{2 n}}{2^{n-2}} \int_{0}^{1} d u u \delta_{i_{1} i_{2} \ldots i_{2 n}}^{j j_{2} \ldots j_{2 n}}\left(\delta_{j_{2}}^{i_{2}} K_{i}^{i_{1}}+\delta_{i}^{i_{2}} K_{j_{2}}^{i_{1}}\right) \mathcal{F}_{j_{3} j_{4}}^{i_{3} i_{4}}(u) \\
& \ldots \mathcal{F}_{j_{2 n-1} j_{2 n}}^{i_{2 n-1} i_{2 n}}(u),
\end{aligned}
$$

where

$\mathcal{F}_{k l}^{i j}(u)=R_{k l}^{i j}-\left(u^{2}-1\right)\left(K_{k}^{i} K_{l}^{j}-K_{l}^{i} K_{k}^{j}\right)+\frac{u^{2}}{\ell_{\mathrm{eff}}^{2}} \delta_{k l}^{i j}$.

The coupling constant in this case is

$$
\begin{aligned}
c_{2 n}= & -\frac{1}{16 \pi n G}\left[\int_{0}^{1} d u\left(1-u^{2}\right)^{n-1}\right]^{-1} \\
& \times \sum_{p=1}^{n} \frac{p \alpha_{p}}{(D-2 p) !}\left(-\ell_{\mathrm{eff}}^{2}\right)^{n-p} .
\end{aligned}
$$

The charge in $D$ dimensions is a polynomial of order $N$ in the curvature. In nondegenerate Lovelock theories, this polynomial can be truncated so that it becomes linear in the Weyl tensor [33]

$$
\tau_{i}^{j}=-\frac{\ell_{\mathrm{eff}} \Delta^{(1)}\left(\ell_{\mathrm{eff}}^{-2}\right)}{32 \pi G(D-3)} \delta_{i i_{2} i_{3}}^{j j_{2} j_{3}} W_{j_{2} j_{3}}^{i_{2} i_{3}} .
$$

This expression corresponds to conformal mass for nondegenerate Lovelock AdS gravity [33], as an extension of the concept developed by Ashtekar, Magnon and Das [6,8]. Using the identity $\delta_{i i_{2}}^{j j_{2}} W_{j_{2} j_{3}}^{i_{2} i_{3}}=4 W_{r i}^{r j}$ the charge becomes proportional to the electric part of the Weyl tensor

$$
\tau_{i}^{j}=-\frac{\ell_{\mathrm{eff}}}{8 \pi G} \Delta^{(1)}\left(\ell_{\mathrm{eff}}^{-2}\right) E_{i}^{j},
$$

where in $D$ dimensions

$$
E_{i}^{j}=\frac{1}{D-3} W_{r i}^{r j}
$$

Clearly Eq. (3.11) fails when $\Delta^{(1)}=0$.

When the theory has multiplicity $k$, a power-counting argument indicates that the asymptotic falloff of $\tau_{i}^{j}$ is such that the system has finite global charges. Namely, the bulk metric behaves as in Eq. (2.26), and so $\sqrt{\sigma}=\mathcal{O}\left(r^{D-2}\right)$, $u_{j}=\mathcal{O}(r)$ and $\xi=\mathcal{O}(1)$. Since the charge $Q[\xi]$ is of order $\mathcal{O}(1)$, it implies that $\tau_{i}^{j}$ should be of order $\mathcal{O}\left(1 / r^{D-1}\right)$. At the same time, the charge should be zero for global AdS. Hence it must be a nonlinear expression in the Weyl tensor.

\section{GENERALIZED CONFORMAL MASS}

In this section we manipulate the general formulas (3.5) and (3.7) for $\tau_{i}^{j}$ to make manifest the dependence on the degeneracy conditions at different orders.

\section{A. Even dimensions $D=\mathbf{2 n}$}

The charge density in even dimensions can be conveniently rewritten as 


$$
\begin{aligned}
\tau_{i}^{j}= & \frac{\ell_{\mathrm{eff}}^{2 n-2}}{16 \pi G 2^{n-2}} \delta_{i_{1} \ldots i_{2 n-1}}^{j j_{2} \ldots j_{2 n-1}} K_{i}^{i_{1}} \sum_{p=1}^{n-1} \frac{p \alpha_{p}}{(2 n-2 p) !}\left(\frac{1}{\ell_{\mathrm{eff}}^{2}}\right)^{p-1} R_{j_{2} j_{3}}^{i_{2} i_{3}} \ldots R_{j_{2 p-2} j_{2 p-1}}^{i_{2 p-1} i_{2 p-1}} \\
& \times\left[\left(\frac{1}{\ell_{\mathrm{eff}}^{2}}\right)^{n-p} \delta_{j_{2 p} j_{2 p+1}}^{i_{2 p} i_{2 p+1}} \ldots \delta_{j_{2 n-2} j_{2 n-1}}^{i_{2 n-2} i_{2 n-1}}-(-1)^{n-p} R_{j_{2 p} j_{2 p+1}}^{i_{2 p} i_{2 p+1}} \ldots R_{j_{2 n-2} j_{2 n-1}}^{i_{2 n-2} i_{2 n-1}}\right] .
\end{aligned}
$$

As it is clear from the above formula, the charge is a polynomial of order $n-1$ in the curvature. For a branch with the degeneracy $k$, this can be rearranged in order to express the polynomial as a product of $k$ AdS curvatures times a polynomial $\mathcal{P}(R)$ of order $n-1-k$ in the curvature

$$
\tau_{i}^{j}=\frac{\ell_{\mathrm{eff}}^{2(n-1)}}{16 \pi G 2^{n-2}} \delta_{i_{1} i_{2} \ldots i_{2 k} i_{2 k+1} \ldots i_{2 n} i_{2 n-1}}^{j j_{2} \ldots j_{2} j_{2 k+1} \ldots j_{2 n} j_{2 n-1}} K_{i}^{i_{1}}\left(R_{j_{2} j_{3}}^{i_{2} i_{3}}+\frac{1}{\ell_{\mathrm{eff}}^{2}} \delta_{j_{2} j_{3}}^{i_{2} i_{3}}\right) \times \cdots \cdots \times\left(R_{j_{2 k} j_{2 k+1}}^{i_{2 k} i_{2 k+1}}+\frac{1}{\ell_{\mathrm{eff}}^{2}} \delta_{j_{2 k} j_{2 k+1}}^{i_{2 k} i_{2 k+1}}\right) \mathcal{P}_{j_{2 k+2} \ldots j_{2 n-1}}^{i_{2 k+2} \ldots i_{2 n-1}}(R),
$$

where $\mathcal{P}(R)$ written as in Eq. (A14) of Appendix A with all the indices

$$
\mathcal{P}_{j_{2 k+2} \ldots j_{2 n-1}}^{i_{2 k+2} \ldots i_{2 n-1}}(R)=\sum_{s=0}^{n-k-1} \sum_{p=k}^{k+s} \Delta^{(p)}\left(C_{k+s, p}\right)_{i_{2 k+2} \ldots i_{2 n-2 s-1}}^{j_{2 k+2} \ldots j_{2 n-2 s-1}}\left(R_{j_{2 n-2 s} j_{2 n-2 s+1}}^{i_{2 n-2} i_{2 n-2 s+1}}+\frac{1}{\ell_{\mathrm{eff}}^{2}} \delta_{j_{2 n-2 s} j_{2 n-2 s+1}}^{i_{2 n-2 s} i_{2 n-2 s+1}}\right) \ldots\left(R_{j_{2 n-2} j_{2 n-1}}^{i_{2 n-2} i_{2 n-1}}+\frac{1}{\ell_{\mathrm{eff}}^{2}} \delta_{j_{2 n-2} j_{2 n-1}}^{i_{2 n-1} i_{2 n-1}}\right) .
$$

For a detailed construction of this factorization in even dimensions, see Appendix A 1. The charge has to be evaluated in the asymptotic region where we know that the curvature tensor behaves as in Eq. (2.30). In turn, the extrinsic curvature has the asymptotic form

$$
K_{j}^{i}=-\frac{1}{\ell_{\mathrm{eff}}} \delta_{j}^{i}+\mathcal{O}\left(1 / r^{2}\right)
$$

Furthermore, given the fact that the charge density at large distances is $\tau_{j}^{i}=\mathcal{O}\left(1 / r^{D-1}\right)$, the AdS curvature falloff (2.32) constrains the polynomial $\mathcal{P}=\mathcal{O}(1)$ to leading order. This implies that the Riemann curvature is $R=-\frac{1}{\ell_{\text {eff }}^{2}} \delta^{[2]}$ in the polynomial so that the only nonzero contribution in Eq. (4.3) comes from $s=0, p=k$, corresponding to the coefficient $C_{k k}$ given by

$$
\left(C_{k k}\right)_{j_{2 k+2} \ldots j_{2 n-1}}^{i_{2 k+2} \ldots i_{2 n-1}}=\frac{(-1)^{k-1}}{2(2 n-3) ! \ell_{\mathrm{eff}}^{2(n-2)}} \delta_{j_{2 k+2} j_{2 k+3}}^{i_{2 k+2} i_{2 k+3}} \ldots \delta_{j_{2 n-2} \ldots j_{2 n-1}}^{i_{2 n-2} i_{2 n-1}}
$$

from Eq. (A25) in Appendix A 1. The above reasoning yields

$$
\mathcal{P}_{j_{2 k+2} \ldots j_{2 n-1}}^{i_{2 k+1} \ldots i_{2 n-1}}\left(-\ell_{\mathrm{eff}}^{-2} \delta^{[2]}\right)=-\frac{(-1)^{k} \Delta^{(k)}}{2(2 n-3) ! \ell_{\mathrm{eff}}^{2(n-2)}} \delta_{j_{2 k+2} j_{2 k+3}}^{i_{2 k+2} i_{2 k+3}} \ldots \delta_{j_{2 n} j_{2 n-1}}^{i_{2} i_{2 n-1}} \cdot
$$

Clearly, this quantity is nonvanishing only in a theory whose vacuum has multiplicity $k$. Replacing the asymptotic form of the extrinsic curvature of Eq. (4.4) and the relation between the AdS curvature and the Weyl tensor of Eq. (2.36), we find that the charge density tensor in even dimensions can be consistently truncated up to the order $k$ in the Weyl tensor,

$$
\tau_{i}^{j}=\frac{\ell_{\mathrm{eff}}(-1)^{k} \Delta^{(k)}}{16 \pi G 2^{n-1}(2 n-3) !} \delta_{i i_{2} \ldots i_{2 k} i_{2 k+1} \ldots j_{2 n} i_{2 n-1}}^{j j_{2} \ldots j_{2 n} j_{2 k+1}} W_{j_{2} j_{3}}^{i_{2} i_{3}} \ldots W_{j_{2 k} j_{2 k+1}}^{i_{2 k} i_{2 k+1}} \delta_{j_{2 k+2} j_{2 k+3}}^{i_{2 k+2} i_{2 k+3}} \ldots \delta_{j_{2 n} j_{2 n-1}}^{i_{2 n} i_{2 n-1}}
$$

Upon a suitable contraction of the Kronecker deltas, the charge becomes

$$
\tau_{i}^{j}=\frac{\ell_{\mathrm{eff}}(-1)^{k} \Delta^{(k)}(2 n-2 k-2) !}{16 \pi G 2^{k}(2 n-3) !} \delta_{i i_{2} \ldots i_{2 k} i_{2 k+1}}^{j j_{2} \ldots j_{2 k} j_{2 k+1}} W_{j_{2} j_{3}}^{i_{2} i_{3}} \ldots W_{j_{2 k} j_{2 k+1}}^{i_{2 k} i_{2 k+1}}
$$

For $k=1$, the last formula reduces to the conformal mass (3.11) in nondegenerate Lovelock theories. In the maximally degenerate case, i.e., Born-Infeld AdS gravity, the charge is a product of $n-1$ Weyl tensors or AdS curvatures, which matches with the result in Ref. [34]. The degeneracy condition (2.9) as an overall factor shows the validity of the formula for a particular gravity theory with multiplicity $k$. Indeed, for theories with the intermediate degeneracy $1<k<n-1$, we can drop $n-k-1$ curvatures from the original charge formula (3.5). 


\section{B. Odd dimensions $D=2 n+1$}

In odd dimensions, black hole mass is associated with $\tau_{i}^{j}$ given by Eq. (3.7), which can be recast as

$$
\begin{aligned}
\tau_{i}^{j}= & \frac{(2 n-1) !\left(-\ell_{\mathrm{eff}}^{2}\right)^{n-1}}{2^{3 n-4}(n-1) !^{2} 16 \pi G} \delta_{i_{1} \ldots i_{2 n}}^{j j_{2} \ldots j_{2 n}} K_{i}^{i_{1}} \delta_{j_{2}}^{i_{2}} \\
& \times \sum_{p=1}^{n} \frac{(-1)^{p} p \alpha_{p} \ell_{\mathrm{eff}}^{2(1-p)}}{(2 n-2 p+1) !} \int_{0}^{1} d u\left(\mathcal{I}_{p}\right)_{j_{3} \ldots j_{2 n}}^{i_{3} \ldots i_{2 n}}(u),
\end{aligned}
$$

where the tensorial quantity $\mathcal{I}_{p}(u)$ is a polynomial of order $n-1$ in the curvature,

$$
\begin{aligned}
\mathcal{I}_{p}(u)= & \left(R+\frac{u^{2}}{\ell_{\mathrm{eff}}^{2}} \delta^{[2]}\right)^{n-1} \\
& -\left(u^{2}-1\right)^{n-1}(-R)^{p-1}\left(\frac{1}{\ell_{\mathrm{eff}}^{2}} \delta^{[2]}\right)^{n-p} .
\end{aligned}
$$

We will restrict our analysis to the theories with $k<n$. We can rearrange the expression for $\tau_{i}^{j}$ and factorize it in a similar fashion as in the even dimensional case (see Appendix A 2),

$$
\begin{aligned}
\tau_{i}^{j}= & \frac{2^{2 n-2}(n-1) !{ }^{2} \ell_{\mathrm{eff}}^{2(n-1)}}{2^{n-2}(2 n-1) ! 16 \pi G} \delta_{i_{1} i_{2} \ldots i_{2 k} i_{2 k+1} \ldots i_{2 n} i_{2 n-1}}^{j j_{2} \ldots j_{2} j_{2 k+1} \ldots j_{2 n} j_{2 n-1}} K_{i}^{i_{1}} \\
& \times\left(R_{j_{2} j_{3}}^{i_{2} i_{3}}+\frac{1}{\ell_{\mathrm{eff}}^{2}} \delta_{j_{2} j_{3}}^{i_{2} i_{3}}\right) \times \cdots \\
& \cdots \times\left(R_{j_{2 k} j_{2 k+1}}^{i_{2 k} i_{2 k+1}}+\frac{1}{\ell_{\mathrm{eff}}^{2}} \delta_{j_{2 k} j_{2 k+1}}^{i_{2 i_{2} i_{2 k+1}}}\right) \mathcal{P}_{j_{2 k+2} \ldots j_{2 n-1}}^{i_{2 k+2} \ldots i_{2 n-1}}(R),
\end{aligned}
$$

where $\mathcal{P}(R)$ is a polynomial in the curvature tensor of order $n-k-1$. It turns out that, again, it has the form of a linear combination of the derivatives $\Delta^{(p)}$ of the characteristic polynomial [see Eq. (A14) in Appendix A]

$$
\begin{aligned}
& \mathcal{P}_{j_{2 k+2} \ldots j_{2 n-1}}^{i_{2 k+1} \ldots i_{2 n-1}}(R) \\
& =\sum_{s=0}^{n-1-k} \sum_{p=k}^{k+s} \Delta^{(p)}\left(C_{k+s, p}\right)_{j_{2 k+2} \ldots j_{2 n-1}}^{i_{2 k+2} \ldots i_{2 n-1}} F_{j_{2 n-2} j_{2 n-1}}^{i_{2 n-1} i_{2 n-1}} \ldots F_{j_{2 n-2} j_{2 n-1}}^{i_{2 n-2} i_{2 n-1}},
\end{aligned}
$$

with some tensorial coefficients $C_{k+s, p}$ that differ from the even dimensional case. Evaluating the polynomial in the asymptotic region, the only part relevant for the conserved charge is

$$
\mathcal{P}_{j_{2 k+2} \ldots j_{2 n-1}}^{i_{2 k+2} \ldots i_{2 n-1}}\left(-\ell_{\text {eff }}^{-2} \delta^{[2]}\right)=\Delta^{(k)}\left(C_{k k}\right)_{j_{2 k+2} \ldots j_{2 n-1}}^{i_{2 k+1} \ldots i_{2 n-1}}
$$

where the coefficient has the form

$$
\begin{aligned}
\left(C_{k k}\right)_{j_{2 k+2} \ldots j_{2 n-1}}^{i_{2 k+2} \ldots i_{2 n-1}}= & \frac{(-1)^{n-k-1} 2^{2 n-3}(n-1) !^{2}}{(2 n-1) !} \ell_{\mathrm{eff}}^{2} \\
& \times \delta_{j_{2 k+2} j_{2 k+3}}^{i_{2 k+2} i_{2 k+3}} \ldots \delta_{j_{2 n} j_{2 n-1}}^{i_{2 n} i_{2 n-1}}
\end{aligned}
$$

as shown in Eq. (A40) of Appendix A 2. Knowing the behavior of the extrinsic curvature (4.4) and the relation (2.36), we can evaluate the charge asymptotically as

$$
\begin{aligned}
\tau_{i}^{j}= & \frac{\ell_{\text {eff }}(-1)^{k} \Delta^{(k)}}{16 \pi G 2^{n-1}(2 n-2) !} \delta_{i i_{2} \ldots i_{2 k} i_{2 k+1} \ldots i_{2 n} i_{2 n-1}}^{j_{2} \ldots j_{2 k} j_{2 k+1} \ldots j_{2 n} j_{2 n-1}} W_{j_{2} j_{3}}^{i_{2} i_{3}} \ldots W_{j_{2 k} j_{2 k+1}}^{i_{2 k} i_{2 k+1}} \\
& \times \delta_{j_{2 k+2} j_{2 k+3}}^{i_{2 k+2} i_{2 k+3}} \ldots \delta_{j_{2 n} j_{2 n-1}}^{i_{2} i_{2 n-1}} .
\end{aligned}
$$

Finally, contracting the antisymmetric deltas, the charge density becomes

$\tau_{i}^{j}=\frac{\ell_{\mathrm{eff}}(-1)^{k} \Delta^{(k)}(2 n-2 k-1) !}{16 \pi G 2^{k}(2 n-2) !} \delta_{i i_{2} \ldots i_{2 k} i_{2 k+1}}^{j j_{2} \ldots j_{2 k} j_{2 k+1}} W_{j_{2} j_{3}}^{i_{2} i_{3}} \ldots W_{j_{2 k} j_{2 k+1}}^{i_{2 k} i_{2 k+1}}$.

The last expression is valid for theories whose AdS vacua have a degeneracy level in the interval $1 \leq k \leq n-1$. It is a generalization of the Ashtekar-Magnon-Das conformal mass formula [6,8] to the Lovelock AdS gravity [33]. Once again, for $k=1$ it reduces to the known conformal mass (3.11).

In the case of Chern-Simons AdS gravity, the mass term does not fall off as $r \rightarrow \infty$. The fact that the energy for global AdS is not continuously connected with the spectrum of black holes of the theory indicates that the energy for the vacuum state cannot be achieved by charge that is proportional to the Weyl tensor. This presents a qualitative difference between the theories with degeneracy $k<n$ and Chern-Simons AdS gravity, which is reflected in their holographic properties $[35,36]$.

\section{Energy of the LUV AdS black hole}

As an example, we calculate the total energy of the LUV AdS black hole solution described by the metric (2.13) with the metric function (2.27). We can identify $\frac{\mu}{\Delta^{(k)}}=2 G M$. Because the tensor $X_{\alpha \beta}^{\mu \nu}$ given by (2.35) identically vanishes in this case, the Weyl tensor and the AdS curvature are equal on-shell, $W_{\alpha \beta}^{\mu \nu}=F_{\alpha \beta}^{\mu \nu}$. The degeneracy (2.9) for the LUV gravity becomes

$$
\Delta_{\mathrm{LUV}}^{(k)}=2^{k-1}(-1)^{k-1}(D-3) ! .
$$

The total energy of the system is the Noether charge (3.4) for the time translations $\xi=\partial_{t}$ and the boundary with the unit vector $u_{j}=-\sqrt{f} \delta_{j}^{t}$. Furthermore, we have $\sigma_{n m}=$ $r^{2} \gamma_{n m}(y)$, where the transverse metric $\gamma_{n m}$ depends only on the transverse coordinates $y^{m}$. Then we can find the 
Jacobian $\sqrt{\sigma}=r^{D-2} \sqrt{\gamma}$ and the volume of the transverse section $\Omega_{D-2}=\int_{\Sigma_{\infty}} d^{D-2} y \sqrt{\gamma}$.

With this at hand, we can evaluate the total energy as

$$
E=Q\left[\partial_{t}\right]=E_{\text {vacum }}-\Omega_{D-2}\left(r^{D-2} \sqrt{f} \tau_{t}^{t}\right)_{r \rightarrow \infty},
$$

where the vacuum energy for Lovelock gravity (the energy of the global AdS space) exists in odd dimensions $D$ only, and was calculated in Ref. [26].

On the other hand, the charge density tensor $\tau_{i}^{j}$ given by Eqs. (A29) and (A44) in even and odd dimensions, respectively, has the form

$\tau_{t}^{t}=\frac{(-1)^{k} \ell_{\mathrm{eff}} \Delta^{(k)}(D-2 k-2) !}{16 \pi G 2^{k}(D-3) !} \delta_{n_{1} \ldots n_{2 k}}^{m_{1} \ldots m_{2 k}} W_{m_{1} m_{2}}^{n_{1} n_{2}} \ldots W_{m_{2 k-1} m_{2 k}}^{n_{2 k-1} m_{2 k}}$.

The only relevant components of the Weyl tensor read

$$
W_{m_{1} m_{2}}^{n_{1} n_{2}}=\left(\frac{2 G M}{r^{D-1}}\right)^{\frac{1}{k}} \delta_{m_{1} m_{2}}^{n_{1} n_{2}}
$$

Using the expression

$\delta_{n_{1} \ldots n_{2 k}}^{m_{1} \ldots m_{2 k}} W_{m_{1} m_{2}}^{n_{1} n_{2}} \ldots W_{m_{2 k-1} m_{2 k}}^{n_{2 k-1} n_{2 k}}=\frac{2 G M}{r^{D-1}} \frac{2^{k}(D-2) !}{(D-2 k-2) !}$,

it is straightforward to evaluate the energy density as

$$
\tau_{t}^{t}=-\frac{\ell_{\mathrm{eff}} 2^{k}(D-2) !}{32 \pi G} \frac{2 G M}{r^{D-1}}
$$

and the total energy of the system

$$
E=E_{\text {vacuum }}+\frac{(D-2) ! 2^{k} \Omega_{D-2}}{16 \pi} M .
$$

The total mass, $E-E_{\text {vacuum }}$, is indeed linear in the parameter $M$. Furthermore, in order to have the charge that is directly $M$ as in Ref. [25], the gravitational action (2.1), (2.12) has to be normalized suitably, by dividing it by $\Omega_{D-2}$, a $D$-dependent factor and introducing the gravitational constant $G_{k}$, which explicitly depends on the multiplicity [37]. General relativity $(k=1)$ and BI gravity $(k=n-1)$ are particular cases of LUV gravity in AdS space.

\section{CONCLUSIONS}

We have derived the conformal mass formula for a branch of Lovelock AdS gravity with a $k$-fold vacuum degeneracy. This comes as the generalization of the results found in Ref. [33], which indicate that the energy of black holes in that theory cannot be written as a linear expression in the AdS curvature (2.31) or, equivalently, in terms of the electric part of the Weyl tensor. On the contrary, we find that the conserved quantity must be nonlinear in the Weyl tensor in order to capture the falloff properties of the mass term in the metric.

To summarize, the charge density tensor has the form

$\tau_{i}^{j}=\frac{(-1)^{k} \ell_{\mathrm{eff}} \Delta^{(k)}(D-2 k-2) !}{16 \pi G 2^{k}(D-3) !} \delta_{i i_{2} \ldots i_{2 k} i_{2 k+1}}^{j j_{2} \ldots j_{2 k} j_{2 k+1}} W_{j_{2} j_{3}}^{i_{2} i_{3}} \ldots W_{j_{2 k} j_{2 k+1}}^{i_{22} i_{2 k+1}}$

in both even and odd dimensions, respectively, given by Eqs. (A29) and (A44). As an example, we showed that this formula gives the total energy of a static topological black hole in AdS space of multiplicity $k$.

When $k=1$, this tensor becomes proportional to the electric part of the Weyl tensor (3.11). In general, when $k>1$, the appearance of the degeneracy condition in the corresponding surface terms coming from an arbitrary variation of the renormalized action (3.1) may be useful in understanding holographic properties of degenerate AdS gravity theories. In particular, it has been claimed $[19,38]$ that the $C_{T}$ coefficient in holographic two-point functions is proportional to the first degeneracy condition (2.8). As the coefficient is linked to the $a$-charge (type $A$-anomaly), recent results suggest that it is necessary to consider higher degeneracy conditions [39] when one deals with theories with degenerate AdS vacua.

\section{ACKNOWLEDGMENTS}

G. A. H. and R. O. thank G. Anastasiou, I. J. Araya, C. Arias, P. Bueno, F. Diaz and D. Rivera-Betancour for insightful comments. This work was supported in part by Chilean FONDECYT Projects No. 1170765 "Boundary dynamics in anti-de Sitter gravity and gauge/gravity duality" and No. 1190533 "Black holes and asymptotic symmetries" and in part by the Natural Sciences and Engineering Research Council of Canada.

\section{APPENDIX A: FACTORIZATION OF THE CHARGE POLYNOMIAL}

In this section we use the following shorthand notation. $\delta^{[p]}$ corresponds to the antisymmetric Kronecker delta of rank $p$, which is, $\delta_{i_{1} \ldots i_{p}}^{j_{1} \ldots j_{p}}$, and $\delta_{i[p]}^{j[p]}$ is a totally antisymmetric Kronecker delta of rank $p$ with the indices $i$ and $j$ fixed, what means $\delta_{i i_{2} \ldots i_{p}}^{j j_{2} \ldots j_{p}}$. Writing out the contracted indices will be omitted.

In both even $D=2 n$ and odd $D=2 n+1$ dimensions, the charge density tensor has the form

$$
\tau_{i}^{j}=\frac{\theta_{D}}{16 \pi G} \delta_{i_{1} \ldots i_{2 n-1}}^{j j_{2} \ldots j_{2 n-1}} K_{i}^{i_{1}} f_{j_{2} \ldots j_{2 n-1}}^{i_{2} \ldots i_{2 n-1}}(R),
$$

or shortly 


$$
\tau_{j}^{i}=\frac{\theta_{D}}{16 \pi G} \delta^{[2 n-1] j} K_{i} f(R),
$$

where the expressions for the particular coefficient $\theta_{D}$ and the auxiliary function $f(R)$, which is the polynomial of order $n-1$ the Riemann curvature, depends on the regularization method, as given by Eqs. (3.5) and (3.7), respectively.

Let the Lovelock coefficients $\alpha_{p}$ be such that the theory has a degenerate $\mathrm{AdS}$ vacuum of order $k$ given through the criterion (2.9). Then our main goal is to show that the function $f$ can be factorized by $\left(R+\frac{1}{\ell_{\mathrm{eff}}^{2}} \delta^{[2]}\right)^{k}$, multiplied by a polynomial $\mathcal{P}$ of the order $n-k-1$ in the Riemann curvature, which is finite in the asymptotic region, and moreover it is proportional to the degeneracy factor $\Delta^{(k)}$.

Thus, we will prove that, in any dimension, the auxiliary function can be put into the form

$$
\begin{aligned}
f_{j_{2} \ldots j_{2 n-1}}^{i_{2} \ldots i_{2 n-1}}(R)= & \left(R_{j_{2} j_{3}}^{i_{2} i_{3}}+\frac{1}{\ell_{\mathrm{eff}}^{2}} \delta_{j_{2} j_{3}}^{i_{2} i_{3}}\right) \\
& \ldots\left(R_{j_{2 k} j_{2 k+1}}^{i_{2 k} i_{2 k+1}}+\frac{1}{\ell_{\mathrm{eff}}^{2}} \delta_{j_{2 k} j_{2 k+1}}^{i_{2 k} i_{2 k+1}}\right) \mathcal{P}_{j_{2 k+2} \ldots j_{2 n-1}}^{i_{2 k+2} \ldots i_{2 n-1}}(R) .
\end{aligned}
$$

Mathematically, these statements are easier formulated if we write $A_{k l}^{i j}=\frac{1}{\ell_{\text {eff }}^{2}} \delta_{k l}^{i j}$, or symbolically

$$
A=\frac{1}{\ell_{\mathrm{eff}}^{2}} \delta^{[2]}
$$

so that we have to prove

$$
f=(A+R)^{k} \mathcal{P}(R),
$$

where the polynomial $\mathcal{P}(R)$ of the order $n-k-1$ cannot be further factorized by $A+R$. Furthermore, $\mathcal{P}$ is finite when $R=-A$, taking the value

$$
\mathcal{P}(-A)=C_{k k} \Delta^{(k)} \neq 0 .
$$

Because the tensor $f_{j_{2} \ldots j_{2 n-1}}^{i_{2} \ldots i_{2 n-1}}$ has $2 n-2$ pairs of indices, and the tensor $\mathcal{P}_{j_{2 k+2} \ldots j_{2 n-1}}^{i_{2 k+2} \ldots i_{2 n-1}}$ has $2 n-2-2 k$ pairs of indices, it implies that $C_{k k}$ has the same index structure as $\mathcal{P}$, that is, $\left(C_{k k}\right)_{j_{2 k+2} \ldots j_{2 n-1}}^{i_{2 k+2} \ldots i_{2 n-1}}$.

The degeneracy condition (2.9) can be written in a simpler way as

$$
\Delta^{(q)}=\sum_{p=q}^{n-1}\left(\begin{array}{l}
p \\
q
\end{array}\right) \ell_{\mathrm{eff}}^{2(q-p)} \beta_{p}
$$

where the new coefficients $\beta_{p}$ collect, along the Lovelock couplings $\alpha_{p}$, also all other dimensionless factors,

$$
\beta_{p}=\frac{(-1)^{p-1}(D-3) ! \alpha_{p}}{(D-2 p-1) !}
$$

The method to prove the formula (A5) is a powerexpansion of the function $f$ in the variable $F=A+R=\frac{1}{\ell_{\mathrm{eff}}^{2}} \delta^{[2]}+R$, which is nothing but the AdS curvature. When expanded, the auxiliary function becomes

$$
f=\sum_{q=1}^{n-1} f_{q} F^{q} .
$$

An explicit expression for $f_{q}$ depends on the dimension. Remarkably, in any dimension, $f_{q}$ is a linear combination of the degeneracy coefficients $\Delta^{(p)}$,

$$
f_{q}=\sum_{p=1}^{q} C_{q p} \Delta^{(p)},
$$

where $C_{q p}$ are constant tensors. The last formula is the key step in finding a generalized conformal mass, because in general it is not straightforward to prove that only the degeneracy conditions $\Delta^{(p)}$ will appear in above expansion.

Equation (A10) is always fullfiled in Lovelock theory. In particular, in the $k$-fold degenerate theories, all $\Delta^{(p)}$ vanish up to the order $k-1$ by definition, implying that

$$
f_{1}=\cdots=f_{k-1}=0
$$

In turn, the lower limit in the sum (A10) changes to

$$
f_{q}=\sum_{p=k}^{q} C_{q p} \Delta^{(p)},
$$

and the first nonvanishing term in the auxiliary function (A9) becomes

$f(R)=\sum_{q=k}^{n-1} \sum_{p=k}^{q} C_{q p} \Delta^{(p)} F^{q}=C_{k k} \Delta^{(k)} F^{k}+\mathcal{O}\left(F^{k+1}\right)$.

It leads to the polynomial

$$
\begin{aligned}
\mathcal{P}(R) & =\sum_{q=k}^{n-1} \sum_{p=k}^{q} C_{q p} \Delta^{(p)} F^{q-k} \\
& =\sum_{s=0}^{n-1-k} \sum_{p=k}^{k+s} C_{k+s, p} \Delta^{(p)} F^{s}=C_{k k} \Delta^{(k)}+\mathcal{O}(F) .
\end{aligned}
$$

It is important to point out that, in general, the polynomial on the global AdS space, $F=R+A=0$, is finite, and its contribution in any $D$ is 


$$
\mathcal{P}(-A)=C_{k k} \Delta^{(k)} \neq 0 .
$$

On the other hand, on the asymptotic boundary, the extrinsic curvature and the AdS tensor behave as Eqs. (4.4) and (2.36), respectively, so that the charge density tensor at large distances becomes

$$
\begin{aligned}
\tau_{j}^{i} & =\frac{\theta_{D}}{16 \pi G} \delta^{[2 n-1] j} K_{i} F^{k} \mathcal{P}(R) \\
& =-\frac{\theta_{D}}{16 \pi G \ell_{\mathrm{eff}}} \delta_{i}^{[2 n-1] j} F^{k} \mathcal{P}(-A)+\mathcal{O}\left(1 / r^{D+1}\right),
\end{aligned}
$$

where $\delta^{[2 n-1] j} \delta_{i}=\delta_{i}^{[2 n-1] j}$. Then, clearly, because $F^{k}$ behaves as $F^{k} \sim \mathcal{O}\left(\mu / r^{D-1}\right)$, it implies that $\tau_{j}^{i} \sim$ $\mathcal{O}\left(\mu / r^{D-1}\right)$ and therefore $Q[\xi] \sim \mathcal{O}(\mu)$ will give exactly the mass by means of the charge formula (3.4). This implies that $\tau_{j}^{i}$ can be consistently truncated in any dimension by leaving only the leading order of $F^{k}=W^{k}+\mathcal{O}\left(1 / r^{D+1}\right)$ in the above formula, and all other $\mathcal{O}\left(1 / r^{D+1}\right)$ terms can be safely dropped out, such that

$$
\tau_{j}^{i}=-\frac{\theta_{D} C_{k k} \Delta^{(k)}}{16 \pi G \ell_{\mathrm{eff}}} \delta_{i}^{[2 n-1] j} W^{k} .
$$

Remarkably, the result is always proportional to $\Delta^{(k)}$, and since $\theta_{D}$ is known, we only have to find the coefficient $C_{k k}$ in a particular dimension, to be able to carry out the charge evaluation until the end. In any case, it must be of the form

$$
\tau_{i}^{j} \propto \frac{\Delta^{(k)}}{16 \pi G} \delta_{i i_{2} \ldots i_{2 k} i_{2 k+1}}^{j j_{2} \ldots j_{2 k} j_{2 k+1}} W_{j_{2} j_{3}}^{i_{2} i_{3}} \ldots W_{j_{2 k} j_{2 k+1}}^{i_{2} i_{2 k+1}}
$$

multiplied by some $D$-dependent real number.

Since the value of $C_{k k}$ strongly depends on the dimension, let us find now the explicit expressions for $f(R)$ and $\mathcal{P}(R)$ in case of the even and odd $D$.

\section{Even dimensions}

Consider the charge density tensor (4.1) in $D=2 n$ dimensions of the form (A2), where the scalar coefficient is

$$
\theta_{2 n}=\frac{\ell_{\mathrm{eff}}^{2(n-1)}}{2^{n-2}},
$$

and the auxiliary function reads

$$
f=\sum_{p=1}^{n-1} \frac{p \alpha_{p} \ell_{\mathrm{eff}}^{2(1-p)} R^{p-1}}{(2 n-2 p) !}\left[\left(\frac{1}{\ell_{\mathrm{eff}}^{2}} \delta^{[2]}\right)^{n-p}-(-R)^{n-p}\right] .
$$

In terms of the variables $A$ and $R$ given by Eq. (A4) and redefined Lovelock coefficients (A8), the function can also be written as

$$
\begin{aligned}
f= & \frac{1}{2(2 n-3) !} \sum_{p=1}^{n-1} \frac{p \beta_{p} \ell_{\mathrm{eff}}^{2(1-p)}}{n-p} \\
& \times\left[(A-F)^{p-1} A^{n-p}-(A-F)^{n-1}\right] .
\end{aligned}
$$

The binomials $(A-F)^{p-1}$ and $(A-F)^{n-1}$ can be expanded in series,

$$
\begin{aligned}
f= & \frac{1}{2(2 n-3) !} \sum_{p=1}^{n-1} \frac{p \beta_{p} \ell_{\mathrm{eff}}^{2(1-p)}}{n-p}\left(\sum_{q=1}^{p-1} \frac{(-1)^{q}(p-1) !}{q !(p-1-q) !}\right. \\
& \left.-\sum_{q=1}^{n-1} \frac{(-1)^{q}(n-1) !}{q !(n-1-q) !}\right) F^{q} A^{n-1-q} .
\end{aligned}
$$

In the above expression it was used that, inside the brackets, the terms $q=0$ cancel out,

$$
\left(\frac{(p-1) !}{(p-1) !}-\frac{(n-1) !}{(n-1) !}\right) A^{n-1}=0,
$$

and the sums start from $q=1$.

Obtained power-series in $F$ has the form (A9), but we also have to show that the corresponding coefficients can be written as a linear combination of the degeneracy parameters $\Delta^{(q)}$ defined in Eq. (A7), as given by Eq. (A10).

Indeed, the explicit form of the first few terms of the series is

$$
\begin{aligned}
f= & \frac{1}{2(2 n-3) !} \sum_{p=1}^{n-1} p \beta_{p} \ell_{\mathrm{eff}}^{2(1-p)}\left(F A^{n-2}-\frac{p+n-3}{2} F^{2} A^{n-3}\right. \\
& \left.+\frac{p^{2}+n p-6 p+n^{2}-6 n+11}{6} F^{3} A^{n-4}+\mathcal{O}\left(F^{4}\right)\right) .
\end{aligned}
$$

Using the definition (A7),

$$
q ! \Delta^{(q)} \ell_{\mathrm{eff}}^{2(1-q)}=\sum_{p=q}^{n-1} p(p-1) \ldots(p-q+1) \ell_{\mathrm{eff}}^{2(1-p)} \beta_{p},
$$

the auxiliary function becomes

$$
\begin{aligned}
f= & \frac{1}{2(2 n-3) !}\left[\Delta^{(1)} F A^{n-2}-\left(\frac{n-2}{2} \Delta^{(1)}\right.\right. \\
& \left.+\ell_{\mathrm{eff}}^{-2} \Delta^{(2)}\right) F^{2} A^{n-3}+\left(\frac{n^{2}-5 n+5}{6} \Delta^{(1)}\right. \\
& \left.\left.+\frac{n-3}{3} \ell_{\mathrm{eff}}^{-2} \Delta^{(2)}+\ell_{\mathrm{eff}}^{-4} \Delta^{(3)}\right) F^{3} A^{n-4}+\mathcal{O}\left(F^{4}\right)\right] .
\end{aligned}
$$

In general, for the term $f=C_{q q} \Delta^{(q)} F^{q}+\cdots$, it can be shown that the corresponding coefficient is 


$$
C_{q q}=\frac{(-1)^{q-1} \ell_{\mathrm{eff}}^{2(1-q)} A^{n-1-q}}{2(2 n-3) !}=\frac{(-1)^{q-1} \delta^{[2] n-1-q}}{2(2 n-3) ! \ell_{\mathrm{eff}}^{2(n-2)}}
$$

Evaluated in the charge density tensor (A17), it gives

$$
\tau_{j}^{i}=\frac{(-1)^{k} e_{\mathrm{eff}} \Delta^{(k)}}{16 \pi G 2^{n-1}(2 n-3) !} \delta_{i}^{[2 n-1] j} \delta^{[2] n-1-k} W^{k},
$$

or equivalently, with all the indices,

$$
\begin{aligned}
\tau_{i}^{j}= & \frac{(-1)^{k} \ell_{\text {eff }} \Delta^{(k)}}{16 \pi G 2^{n-1}(2 n-3) !} \delta_{i i_{2} \ldots i_{2 k} i_{2 k+1} \ldots i_{2 n} i_{2 n-1}}^{j j_{2} \ldots j_{2 n} j_{2 k+1} \ldots j_{2 n} j_{2 n-1}} W_{j_{2} j_{3}}^{i_{2} i_{3}} \ldots W_{j_{2 k} j_{2 k+1}}^{i_{2 k} i_{2 k+1}} \\
& \times \delta_{j_{2 k+2} j_{2 k+3}}^{i_{2 k+2} i_{2 k+3}} \ldots \delta_{j_{2 n} j_{2 n-1}}^{i_{2 n} i_{2 n-1}} .
\end{aligned}
$$

By means of the identity

$$
\begin{array}{r}
\delta_{i i_{2} \ldots i_{2 k} i_{2 k+1} \ldots i_{2 n} i_{2 n-1}}^{j j_{2} \ldots j_{2 n} j_{2 n}} \delta_{j_{2 k+2} j_{2 k+3}}^{i_{2 k+2} i_{2 k+3}} \ldots \delta_{j_{2 n} j_{2 n-1}}^{i_{2 n} i_{2 n-1}} \\
=2^{n-k-1}(2 n-2-2 k) ! \delta_{i i_{2} \ldots j_{2 k} j_{2 k+1}}^{j j_{2} \ldots j_{2 k} j_{2 k+1}},
\end{array}
$$

we arrive at the result

$$
\tau_{i}^{j}=\frac{(-1)^{k} \ell_{\mathrm{eff}} \Delta^{(k)}(2 n-2-2 k) !}{16 \pi G 2^{k}(2 n-3) !} \delta_{i i_{2} \ldots i_{2 k} i_{2 k+1}}^{j j_{2} \ldots j_{2 k} j_{2 k+1}} W_{j_{2} j_{3}}^{i_{2} i_{3}} \ldots W_{j_{2 k} j_{2 k+1}}^{i_{2 k} i_{2 k+1}} .
$$

\section{Odd dimensions}

In $D=2 n+1$ dimensions, the charge density tensor (4.9) has the form (A2), with the scalar factor

$$
\theta_{2 n+1}=\frac{(-1)^{n}(2 n-1)}{2^{3 n-4}(n-1) !^{2}}
$$

and the auxiliary function

$$
f=\sum_{p=1}^{n} \frac{p \beta_{p} \ell_{\mathrm{eff}}^{2(n-p)}}{2 n-2 p+1} \int_{0}^{1} d u \mathcal{I}_{p}(u)
$$

The tensorial quantity $\mathcal{I}_{p}(u)$ is given by Eq. (4.10). The goal is to factorize the function $f$ and expand it as a power series in the variable $F=R+A$, as given by Eq. (A4). With these redefinitions, the tensorial quantity becomes

$$
\mathcal{I}_{p}=\left(F-A+u^{2} A\right)^{n-1}-\left(u^{2}-1\right)^{n-1} A^{n-p}(A-F)^{p-1} .
$$

If $F=0$, we have $\mathcal{I}_{p}=0$, as expected. When the binomials $\left(F-A+u^{2} A\right)^{n-1}$ and $(A-F)^{p-1}$ are expanded in series, the integral acquires the form

$$
\begin{aligned}
\mathcal{I}_{p}= & \left(\sum_{q=0}^{n-1} \frac{(n-1) !\left(u^{2}-1\right)^{n-1-q}}{q !(n-1-q) !}\right. \\
& \left.-\sum_{q=0}^{p-1} \frac{(-1)^{q}(p-1) !\left(u^{2}-1\right)^{n-1}}{q !(p-1-q) !}\right) A^{n-1-q} F^{q} .
\end{aligned}
$$

The $q=0$ terms in the sums cancel out exactly, because

$$
\left(\frac{(n-1) !\left(u^{2}-1\right)^{n-1}}{(n-1) !}-\frac{(p-1) !\left(u^{2}-1\right)^{n-1}}{(p-1) !}\right) A^{n-1}=0,
$$

and we can drop them out of the sums. Then the integrations in $u$ can be performed using

$$
\int_{0}^{1} d u\left(u^{2}-1\right)^{n-1}=\frac{(-1)^{n-1} 2^{2 n-2}(n-1) !^{2}}{(2 n-1) !},
$$

giving rise to

$$
\int_{0}^{1} d u \mathcal{I}_{p}=(-1)^{n-1} 2^{2 n-2}(n-1) !\left(\sum_{q=1}^{n-1} \frac{(n-q-1) !}{2^{2 q}(2 n-2 q-1) !}-\frac{(n-1) !}{(2 n-1) !} \sum_{q=1}^{p-1} \frac{(-1)^{q}(p-1) !}{(p-1-q) !}\right) \frac{(-1)^{q}}{q !} A^{n-1-q} F^{q}
$$

Using this result, the auxiliary function becomes

$$
f=(-1)^{n-1} 2^{2 n-2}(n-1) ! \sum_{p=1}^{n} \frac{p \beta_{p} \ell_{\mathrm{eff}}^{2(n-p)}}{2 n-2 p+1}\left(\sum_{q=1}^{n-1} \frac{(-1)^{q}(n-q-1) !}{2^{2 q} q !(2 n-2 q-1) !}-\frac{(n-1) !}{(2 n-1) !} \sum_{q=1}^{p-1} \frac{(-1)^{q}(p-1) !}{q !(p-1-q) !}\right) A^{n-1-q} F^{q} .
$$

Similarly as in the even-dimensional case, the coefficients in the expansion can be rearranged so that they are expressed in terms of the characteristic polynomials $\Delta^{(p)}$, so that $f$ acquires the form (A13). For example, the straightforward evaluation gives the first few terms as

$$
\begin{aligned}
f= & \frac{(-1)^{n} 2^{2 n-3}(n-1) !^{2}}{(2 n-1) !} \sum_{p=1}^{n} p \beta_{p} \ell_{\mathrm{eff}}^{2(n-p)}\left(A^{n-2} F-\frac{2 n+2 p-5}{4} A^{n-3} F^{2}\right. \\
& \left.+\frac{4 p^{2}+4 p n-22 p+4 n^{2}-20 n+33}{24} A^{n-4} F^{3}+\mathcal{O}\left(F^{4}\right)\right) .
\end{aligned}
$$


Rewriting the coefficient in terms of Eq. (A7), we find

$$
\begin{aligned}
f= & \frac{(-1)^{n} 2^{2 n-3}(n-1) !^{2}}{(2 n-1) !}\left[\left[\ell_{\mathrm{eff}}^{2(n-1)} \Delta^{(1)} A^{n-2} F-\left(\ell_{\mathrm{eff}}^{2(n-2)} \Delta^{(2)}+\frac{2 n-3}{4} \ell_{\mathrm{eff}}^{2(n-1)} \Delta^{(1)}\right) A^{n-3} F^{2}\right.\right. \\
& \left.+\left(\ell_{\mathrm{eff}}^{2(n-3)} \Delta^{(3)}+\frac{2 n-5}{6} \ell_{\mathrm{eff}}^{2(n-2)} \Delta^{(2)}+\frac{(2 n-3)(2 n-5)}{24} \ell_{\mathrm{eff}}^{2(n-1)} \Delta^{(1)}\right) A^{n-4} F^{3}+\mathcal{O}\left(F^{4}\right)\right]
\end{aligned}
$$

Even though a general $C_{q p}$ in the expansion (A13) is difficult to find, we need only the coefficient $C_{q q}$, which can be determined as

$$
C_{q q}=\frac{(-1)^{n-q-1} 2^{2 n-3}(n-1) !^{2}}{(2 n-1) !} \ell_{\mathrm{eff}}^{2(n-q)} A^{n-q-1}=\frac{(-1)^{n-q-1} 2^{2 n-3}(n-1) !^{2}}{(2 n-1) !} \ell_{\mathrm{eff}}^{2} \delta^{[2] n-q-1} .
$$

Now it is straightforward to evaluate the charge density tensor given by Eq. (A17),

$$
\tau_{j}^{i}=\frac{(-1)^{k} \ell_{\mathrm{eff}} \Delta^{(k)}}{16 \pi G 2^{n-1}(2 n-2) !} \delta_{i}^{[2 n-1] j} \delta^{[2] n-k-1} W^{k},
$$

what can be written will all indices as

$$
\tau_{j}^{i}=\frac{(-1)^{k} \ell_{\mathrm{eff}} \Delta^{(k)}}{16 \pi G 2^{n-1}(2 n-2) !} \delta_{i i_{2} \ldots i_{2 k} i_{2 k+1} \ldots i_{2 n} i_{2 n-1}}^{j_{2} \ldots j_{2 k} j_{2 k+1} \ldots j_{2} j_{2 n-1}} W_{j_{2} j_{3}}^{i_{2} i_{3}} \ldots W_{j_{2 k} j_{2 k+1}}^{i_{2_{k}} i_{2 k+1}} \delta_{j_{2 k+2} j_{2 k+3}}^{i_{2 k+2} i_{2 k+3}} \ldots \delta_{j_{2 n} j_{2 n-1}}^{i_{2} i_{2 n-1}} .
$$

Using the identity

$$
\delta_{i i_{2} \ldots i_{2 k} i_{2 k+1} \ldots i_{2 n} i_{2 n-1}}^{j j_{2} \ldots j_{2 k} j_{2 k 1}} \delta_{j_{2 k+2} j_{2 k+3}}^{i_{2 k+2} i_{2 k+3}} \ldots \delta_{j_{2 n} j_{2 n-1}}^{i_{2 n} i_{2 n-1}}=2^{n-k-1}(2 n-2 k-1) ! \delta_{i i_{2} \ldots i_{2 k} i_{2 k+1}}^{j_{2} \ldots j_{2 k} j_{2 k+1}}
$$

the charge density becomes

$$
\tau_{j}^{i}=\frac{(-1)^{k} \ell_{\mathrm{eff}} \Delta^{(k)}(2 n-2 k-1) !}{16 \pi G 2^{k}(2 n-2) !} \delta_{i i_{2} \ldots i_{2 k} i_{2 k+1}}^{j j_{2} \ldots j_{2 k} j_{2 k+1}} W_{j_{2} j_{3}}^{i_{2} i_{3}} \ldots W_{j_{2 k} j_{2 k+1}}^{i_{2 k} i_{2 k+1}}
$$

Note that, in the formula (A37) for the auxiliary function, the polynomial in $F$ is only until the order $F^{n-1}$, because the sums in $p$ and $q$ have domains $1 \leq q \leq p-1 \leq n-1$. This implies that the charge density contains the coefficients $\Delta^{(q)}$ only until $\Delta^{(n-1)}$. However, in the ChernSimons theory, all coefficients $\Delta^{(q)}$ vanish, except $\Delta^{(n)} \neq 0$, giving

$$
\tau_{\mathrm{CS}, i}^{j}=0
$$

For that reason, the mass of Chern-Simons AdS black hole comes from the term $\tau_{(0) i}^{j}$, due to presence of the energy gap between the AdS space and the zero mass black hole, as discussed in subsection IV B.
[1] D. Lovelock, The Einstein tensor and its generalizations, J. Math. Phys. (N.Y.) 12, 498 (1971).

[2] B. Zwiebach, Curvature squared terms and string theories, Phys. Lett. 156B, 315 (1985).

[3] C. W. Misner, K. S. Thorne, and J. A. Wheeler, Gravitation (Freeman, San Francisco, 1973).
[4] R. L. Arnowitt, S. Deser, and C. W. Misner, Canonical variables for general relativity, Phys. Rev. 117, 1595 (1960).

[5] R. L. Arnowitt, S. Deser, and C. W. Misner, The dynamics of general relativity, Gen. Relativ. Gravit. 40, 1997 (2008).

[6] A. Ashtekar and A. Magnon, Asymptotically anti-de Sitter space-times, Classical Quantum Gravity 1, L39 (1984). 
[7] V. Balasubramanian and P. Kraus, A stress tensor for antide Sitter gravity, Commun. Math. Phys. 208, 413 (1999).

[8] A. Ashtekar and S. Das, Asymptotically anti-de Sitter spacetimes: Conserved quantities, Classical Quantum Gravity 17, L17 (2000).

[9] S. Das and R. B. Mann, Conserved quantities in Kerr-anti-de Sitter space-times in various dimensions, J. High Energy Phys. 08 (2000) 033.

[10] R. B. Mann and D. Marolf, Holographic renormalization of asymptotically flat spacetimes, Classical Quantum Gravity 23, 2927 (2006).

[11] J. D. Brown and J. W. York, Jr., The microcanonical functional integral. 1. The gravitational field, Phys. Rev. D 47, 1420 (1993).

[12] J. D. Brown, J. Creighton, and R. B. Mann, Temperature, energy and heat capacity of asymptotically anti-de Sitter black holes, Phys. Rev. D 50, 6394 (1994).

[13] J. D. E. Creighton and R. B. Mann, Quasilocal thermodynamics of dilaton gravity coupled to gauge fields, Phys. Rev. D 52, 4569 (1995).

[14] P. L. McGrath, R. J. Epp, and R. B. Mann, Quasilocal conservation laws: Why we need them, Classical Quantum Gravity 29, 215012 (2012).

[15] R. J. Epp, P. L. McGrath, and R. B. Mann, Momentum in general relativity: Local versus quasilocal conservation laws, Classical Quantum Gravity 30, 195019 (2013).

[16] R. Olea, Regularization of odd-dimensional AdS gravity: Kounterterms, J. High Energy Phys. 04 (2007) 073.

[17] G. Kofinas and R. Olea, Universal kounterterms in Lovelock AdS gravity, Fortschr. Phys. 56, 957 (2008).

[18] Z.-Y. Fan, B. Chen, and H. Lu, Criticality in EinsteinGauss-Bonnet gravity: Gravity without graviton, Eur. Phys. J. C 76, 542 (2016).

[19] X. O. Camanho, J. D. Edelstein, and J. M. Sánchez De Santos, Lovelock theory and the AdS/CFT correspondence, Gen. Relativ. Gravit. 46, 1637 (2014).

[20] S. Deser and B. Tekin, Energy in generic higher curvature gravity theories, Phys. Rev. D 67, 084009 (2003).

[21] A. N. Petrov, Field-theoretical construction of currents and superpotentials in Lovelock gravity, arXiv:1903.05500.

[22] D. G. Boulware and S. Deser, String-Generated Gravity Models, Phys. Rev. Lett. 55, 2656 (1985).

[23] R.-G. Cai, Gauss-Bonnet black holes in AdS spaces, Phys. Rev. D 65, 084014 (2002).

[24] R.-G. Cai, A note on thermodynamics of black holes in Lovelock gravity, Phys. Lett. B 582, 237 (2004).

[25] J. Crisostomo, R. Troncoso, and J. Zanelli, Black hole scan, Phys. Rev. D 62, 084013 (2000).
[26] G. Kofinas and R. Olea, Universal regularization prescription for Lovelock AdS gravity, J. High Energy Phys. 11 (2007) 069.

[27] M. Henningson and K. Skenderis, The holographic Weyl anomaly, J. High Energy Phys. 07 (1998) 023.

[28] R. B. Mann, Misner string entropy, Phys. Rev. D 60, 104047 (1999).

[29] S. de Haro, S. N. Solodukhin, and K. Skenderis, Holographic reconstruction of space-time and renormalization in the AdS/CFT correspondence, Commun. Math. Phys. 217, 595 (2001).

[30] R. Olea, Mass, angular momentum and thermodynamics in four-dimensional Kerr-AdS black holes, J. High Energy Phys. 06 (2005) 023.

[31] G. Giribet, O. Miskovic, R. Olea, and D. Rivera-Betancour, Energy in higher-derivative gravity via topological regularization, Phys. Rev. D 98, 044046 (2018).

[32] G. Anastasiou, I. J. Araya, and R. Olea, Topological terms, $\mathrm{AdS}_{2 n}$ gravity and renormalized entanglement entropy of holographic CFTs, Phys. Rev. D 97, 106015 (2018).

[33] G. Arenas-Henriquez, O. Miskovic, and R. Olea, Vacuum degeneracy and conformal mass in Lovelock AdS gravity, J. High Energy Phys. 11 (2017) 128.

[34] O. Miskovic and R. Olea, Counterterms in dimensionally continued AdS gravity, J. High Energy Phys. 10 (2007) 028.

[35] M. Banados, A. Schwimmer, and S. Theisen, Chern-Simons gravity and holographic anomalies, J. High Energy Phys. 05 (2004) 039.

[36] M. Banados, R. Olea, and S. Theisen, Counterterms and dual holographic anomalies in CS gravity, J. High Energy Phys. 10 (2005) 067.

[37] More precisely, the comparison of the notation of Ref. [25] and our procedure is as follows. When the LUV action of this reference $I_{k}$ is rewritten in a tensorial form, we identify our gravitational constant $G$ with their gravitational constant $G_{k}$ through the relation $\frac{1}{16 \pi G}=\frac{\kappa}{2^{k-1}} \equiv \frac{1}{2^{k}(D-2) ! \Omega_{D-2} G_{k}}$. Thus, the black hole solution in our notation has the constant $M G$ and in the considered reference $M_{k} G_{k}$, leading to another identification $M G=M_{k} G_{k}$. From (4.23), therefore, the black hole mass is $E-E_{\text {vacuum }}=M_{k}$.

[38] P. Bueno, P. A. Cano, R. A. Hennigar, and R. B. Mann, Universality of Squashed-Sphere Partition Functions, Phys. Rev. Lett. 122, 071602 (2019).

[39] Y.-Z. Li, H. Lü, and Z.-F. Mai, Universal structure of covariant holographic two-point functions in massless higher-order gravities, J. High Energy Phys. 10 (2018) 063. 